\title{
Ulnar Dimelia - a rare and neglected anomaly of upper extremity
}

\author{
Yasir Salam Siddiqui ${ }^{\text {a, }}$, Mazhar Abbas ${ }^{\text {a }}$, Nusra Rahman ${ }^{\text {b }}$, Julfiqar ${ }^{\text {a }}$ \\ ${ }^{a}$ Department of Orthopaedic Surgery, Faculty of Medicine, J. N. Medical College, A.M.U., Aligarh 202002, India. \\ ${ }^{b}$ Department of Anatomy, Faculty of Medicine, J. N. Medical College, A.M.U., Aligarh 202002, India.
}

\begin{abstract}
Ulnar dimelia is a rare congenital anomaly of the upper extremity. The condition presents with double ulnae forearm and polydactyly with complete absence of radius bone and thumb. Clinically it presents not only as functional deformity but also as cosmetic deformity. The case presented here is of a one-and-a-half-year-old male baby, born with right side ulnar dimelia with no other musculoskeletal defect. We believe that the case presented here deserves reporting not only because of the rarity of the disease but also due to its difficult management requiring multiple staged operations and henceforth consequent neglect on the part of parents. An attempt is also made to do a brief literature review.

Keywords: Ulnar dimelia; polydactyly; ulna; radius; thumb
\end{abstract}

\section{INTRODUCTION}

Ulnar dimelia also referred to as mirror hand syndrome is a rare congenital anomaly of the upper extremity. The condition presents with double ulnae forearm with complete absence of radius bone. There is also polydactyly with absence of thumb ${ }^{[1,2]}$. Along with musculoskeletal deformity, malformation of the neurovascular structures in the form of doubling of the ulnar nerve and artery with absence of the radial artery has also been reported in the literature ${ }^{[1-3]}$. Clinically it presents not only as functional deformity but also as cosmetic deformity. The broadened elbow, short forearm, radially deviated wrist, polydactyly and absent thumb gives a poor unacceptable cosmetic appearance. The functional deficit with ulnar dimelia is restricted elbow range of motion, forearm rotations, and oppositional grasp ${ }^{[4]}$.

Most cases of mirror hand syndrome are sporadic, while few case studies designated associated genetic syndromes ${ }^{[5,6]}$. Ulnar dimelia results from the imbal-

\footnotetext{
* Corresponding author: Yasir Salam Siddiqui

Mailing address: Department of Orthopaedic Surgery, Faculty of Medicine, J. N. Medical College, A.M.U., Aligarh 202002, India.

E-mail: yassu98@gmail.com

Received: 11 December 2020 / Accepted: 12 January 2021
}

ance of the inductive signals across the radio-ulnar plane during embryonic development ${ }^{[3,7]}$. Failure of the morphogenesis signals on the radial side, while the concentration of such signals on the ulnar side produces ulnar dimelia. Diverse variants of the disease depend upon the severity of the disproportion of the signals across the radio ulnar plane ${ }^{[2,8]}$. In literature case studies are reported, highlighting the various aspects of deformity including anatomical variations, clinical presentation, and management ${ }^{[1-4]}$. We believe that the case presented here deserves reporting not only because of the rarity of the disease but also due to its difficult and prolonged management requiring multiple staged operations and henceforth consequent neglect on the part of parents. An attempt is also made to do a brief literature review.

\section{CASE REPORT}

A one-and-a-half-year-old male baby was brought to us by his parents with complaints of restriction of movements of the right elbow with polydactyly, giving an unacceptable cosmetic appearance of the forearm and hand. They further added that the child is also having difficulty in holding the objects. The patient was their third child with two female siblings without any such complaints. There was no history of consanguinity. The baby was full term, delivered through the vaginal 
route. There was no history suggestive of any maternal infection or any exposure to teratogenic agents throughout the antenatal period. There was no family history of the congenital elbow and hand anomalies in first degree lineages. There was no developmental delay. No history of any other joint involvement or other systemic illness. Clinical examination revealed shortening of the right forearm with fixed flexion deformity of 20 degrees at the elbow, with a further 50 degrees of free flexion possible (range of motion $=20$ to 70 degrees). Forearm rotations were grossly restricted. The wrist was deviated radially with the hand having six well-formed fingers in a single plane (Figure 1). Contour and the movements of the right shoulder were comparable to the left shoulder. The child was able to hold the objects in between fingers (crude grasp). However fine grasp requiring opposition function was not conceivable. The left upper extremity was absolutely normal. There were no other skeletal abnormalities. Furthermore, no definite syndromic features were noted. Systemic examination was unremarkable. Ultrasonography of the abdomen and echocardiography were normal. The radiographic anatomical features of our patient as seen in Figure 2 and 3 could be summa-

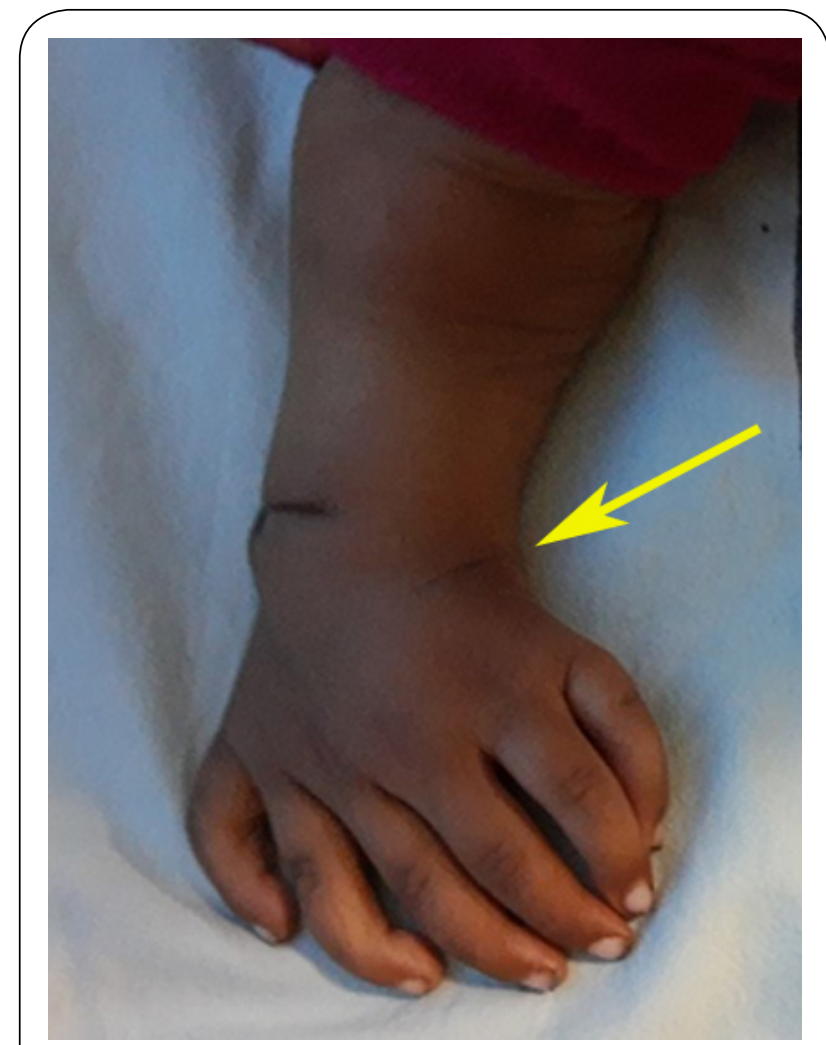

Figure 1. Clinical photograph of patient showing radial deviation of the right wrist (arrow) with the hand having six well-formed fingers in single plane. Also note the absence of thumb.

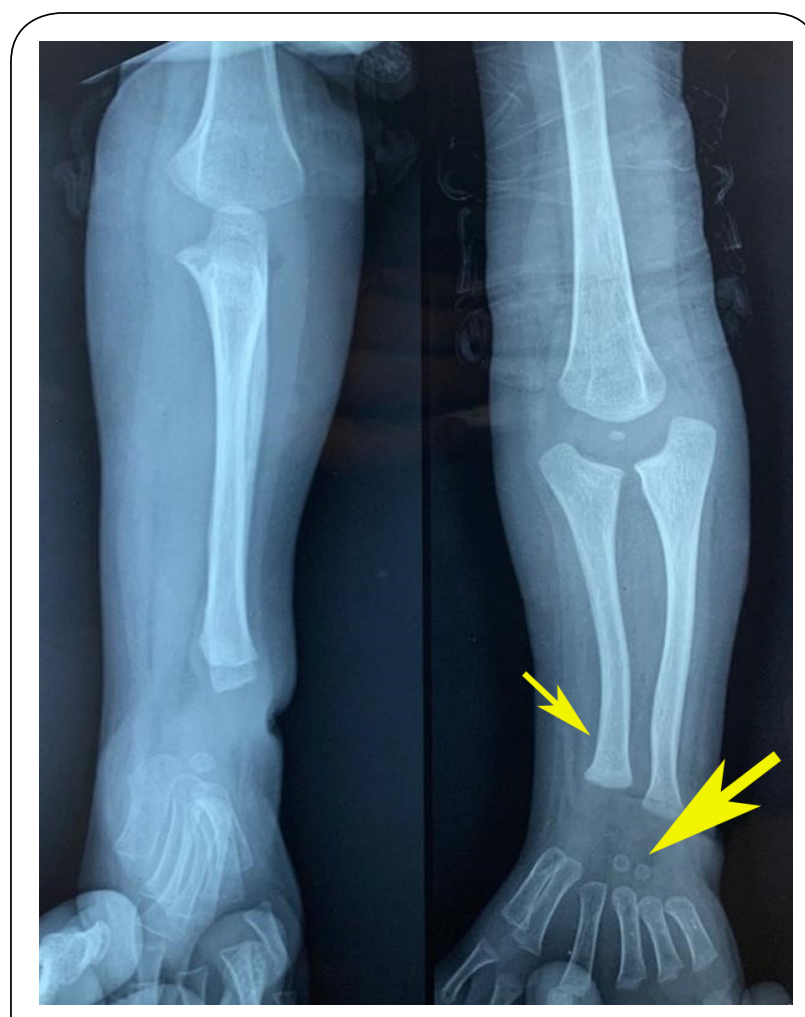

Figure 2. Radiograph of the right upper extremity including elbow revealed double ulnae facing each other with complete absence of radius and thumb. The laterally placed ulna is hypoplastic with a broad distal end (thin arrow). The wrist and hand showed two carpal bones and six metacarpals all lying in the same plane. The two carpal bones were centered over the medially placed ulna (thick arrow).

rized as follows :

(1) A normal humerus with an ossification center in the post-axial part of the distal epiphysis;

(2) Thumb and radius was absent;

(3) Two ulnae facing each other: post-axial ulna had a proper morphology and pre-axial ulna was shorter with less defined proximal anatomical features;

(4) Wrist shows duplication of ossification centers corresponding to lunate;

(5) Normal morphology of metacarpals and phalanges of three post-axial fingers;

(6) Hypoplasia of all pre-axial metacarpals;

(7) Shortening of all phalanges of the pre-axial index finger;

(8) Normal pre-axial middle (M) and ring (R) fingers;

(9) Hypoplasia of pre-axial little (L) finger.

The wrist and hand showed two carpal bones and six metacarpals all lying in the same plane compared to the normal left hand (Figure 2 and 3). The two carpal bones were centered over the medially placed ulna. In the present circumstance, we planned for staged surgeries, with an objective to provide a functional range of motion at the elbow, forearm rotations, and 


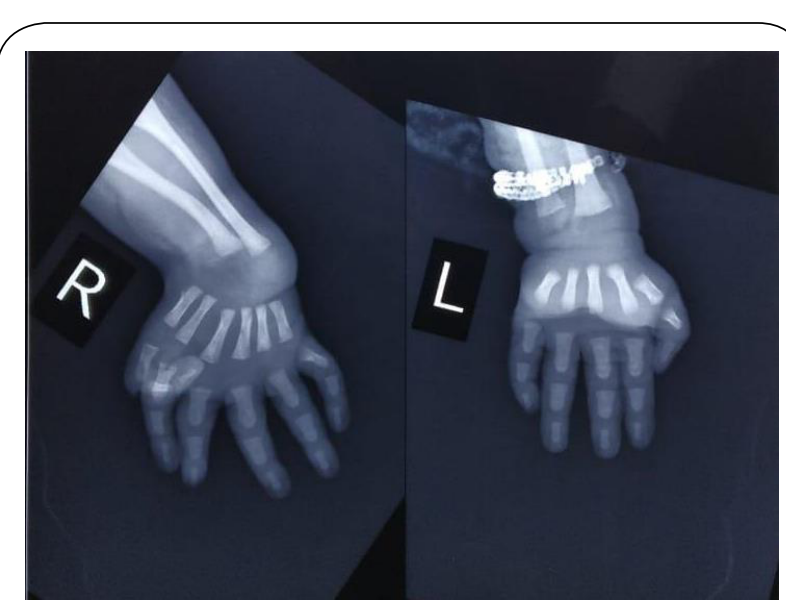

Figure 3. Radiograph of hand showing ulnar dimelia in right hand compared with normal left hand.

reconstruction of the thumb for oppositional grasp. Meanwhile, the patient was put on stretching exercises of elbow, wrist and fingers, especially to correct the radial deviation of the wrist along with night splinting of the wrist in a neutral position. Parents were communicated about the need for regular follow-up for proper treatment and rehabilitation of the child, least as the child would grow there will be limitations of activities which require coordinated movement of the elbow, forearm, and hand-like writing, buttoning, unbuttoning, tying shoelaces and others. However, the parents never turned up again after their first visit, highlighting the neglect of the deformity.

\section{DISCUSSION}

Our patient had double ulnae in the right forearm with six digits with six corresponding metacarpals in a single plane with two carpal bones supported by a medially placed ulna. Laterally placed hypoplastic ulna was not supporting any of the carpal bones. This might be due to a delay in the development of the ossification centers on the lateral side. Both thumb and radius were absent. Although the laterally placed ulna was hypoplastic in comparison to the medial one, both ulnae were well molded. Our case was classified as type I ulnar dimelia, according to Al-Qattan et al classification ${ }^{[2]}$.

Ulnar dimelia is probably due to the failure of differentiation of a part of the ray rather than its pure duplication ${ }^{[18]}$. During the embryogenesis, a disturbance of differentiation of Zone of Polarizing Activity (ZPA) along the axis of the limb may lead to post-axial duplication and ulnar dimelia ${ }^{[3]}$. Few authors have reported HOX genes mutations or translocation breakpoint at $14 \mathrm{q} 13$ as the genetic mechanisms of ulnar-dimelia ${ }^{[19,20]}$. A classical ulnar dimelia has symmetrical distribution around the midline axis. However, the non-classical one lacks symmetrical distribution in morphogenesis of the forearm bones and the fingers ${ }^{[8]}$. In the nonclassical one, the forearm may contain an ulna and a radius, or even three bones in different combinations ${ }^{[9-11]}$. Even though seven ${ }^{[9,10]}$ or eight ${ }^{[2,10-14]}$ digits are typical, patients with six ${ }^{[15,16]}$ or even five digits ${ }^{[17]}$ have been described in the literature. Taking into account the bony morphology of the forearm, Al-Qattan et al published a descriptive classification for the ulnar dimelia ${ }^{[2]}$. The goal of the management of such a condition is to achieve a functional and cosmetically acceptable upper extremity by executing single-stage or more often multiple-stage operations. The literature designates, depending upon the severity of the functional and cosmetic deformity, the management of this condition varies from physiotherapy alone to multiplestage operations ${ }^{[1,2,7,8,11,18]}$. The best age for the reconstruction of hand is before the second year ${ }^{[11]}$. Surgery is preceded by passive range of motion exercises and choosing the appropriate radial digit for pollicising. Hand deformities can be corrected to give a satisfactory outcome when they are not so severe. Either index or middle finger can be pollicised depending upon the type of ulnar dimelia. When deformity correction is not feasible the goal is to achieve the hold function of the hand. Good functional and cosmetic results have been reported in a few studies ${ }^{[3]}$. The thenar reconstruction by interosseous muscle transfers and further improvements can be achieved by tendon transfer at a later date has also been described in the literature ${ }^{[21]}$. The function of the wrist, forearm, and elbow may improve with arthroplasty of the affected joints ${ }^{[22]}$.

In the present case study, we discussed with the parents about the future prospects and limitations of activities due to deformity and planned for staged surgeries with an objective to provide functional and cosmetically acceptable upper extremity. However, the parents never turned up again with the patient for taking treatment, highlighting the neglect of the deformity. The neglect was probably due to the low socioeconomic status and ignorance of parents. We believe that in the patient described above, excision of the proximal end of the laterally placed ulna with soft tissue reconstruction along the medially placed ulna and pollicization would have given a reasonable functional and cosmetic enhancement of the upper extremity. 


\section{CONCLUSION}

Ulnar dimelia is a rare congenital anomaly presenting as functional as well as cosmetic deformity. If untreated, renders the patient with poor function and cosmetic appearance of the extremity. The condition not only has the difficult management requiring multiple staged operations is a therapeutic challenge for treating orthopaedic surgeon but also neglect on the part of caregivers. Hence, awareness among the masses is to be developed regarding the condition and educating them that an acceptable outcome can be achieved with proper treatment.

\section{DECLARATIONS}

\section{Authors' contributions}

Siddiqui YS and Abbas M: substantial contribution to conception and designing and supervision of the study. Nusra Rahman and Julfiqar: provided material \& technical support for the study.

\section{Conflict of interest}

All authors declared that there are no conflicts of interest.

\section{Ethics approval and consent to participate}

Informed consent was obtained from the parents of the patient to participate in the study.

\section{Consent for publication}

Written informed consent for publication was obtained.

\section{REFERENCES}

1. Alosaimi, M. N., Samman, A. T., Alswat, M. M., \& Alsaggaf, K. W. (2020). A rare developmental anomaly of the upper limb: Case of ulnar dimelia. Journal of Musculoskeletal Surgery and Research, 4(3), 160.

2. Al-Qattan, M. M., Al-Thunayan, A., De Cordier, M., Nandagopal, N., \& Pitkanen, J. (1998). Classification of the mirror hand-multiple hand spectrum. Journal of Hand Surgery, 23(4), 534-536.

3. Tomaszewski, R., \& Bulandra, A. (2015). Ulnar dimelia-diagnosis and management of a rare congenital anomaly of the upper limb. journal of orthopaedics, 12, S121-S124.

4. Kay, S. P., McCombe, D., \& Kozin, S. H. (2005). Deformities of the hand and fingers. Green's Operative Hand Surgery (7th Edition)(Eds. Wolfe, Hotchkiss, Pederson, Kozin, Cohen.)(Elsevier, 2016).

5. Sandrow, R. E., Sullivan, P. D., \& Steel, H. H. (1970). Hereditary ulnar and fibular dimelia with peculiar facies: a case report. JBJS, 52(2), 367-370.

6. Martin, R. A., Jones, M. C., \& Jones, K. L. (1993). Mirror hands and feet with a distinct nasal defect, an autosomal dominant condition. American journal of medical genetics, 46(2), 129-131.

7. Gaba, S., John, N., Bhogesha, S., Singh, O., \& Vemula, G. K. (2017). Mirror Hand: An Uncommon Neglected Case Managed with Pollicisation. World journal of plastic surgery, 6(2), 263.

8. Afshar, A. (2010). Ulnar dimelia without duplicated arterial anatomy. The Journal of Bone and Joint Surgery. British volume, 92(2), 293-296.

9. Barton, N. J., Buck-Gramcko, D., \& Evans, D. M. (1986). Soft-tissue anatomy of mirror hand. Journal of Hand Surgery, 11(3), 307-319.

10. Bhaskaranand, K., Bhaskaranand, N., \& Bhat, A. K. (2003). A variant of mirror hand: a case report. The Journal of hand surgery, 28(4), 678-680.

11. Jafari, D., \& Sharifi, B. (2005). A variant of mirror hand: a case report. The Journal of Bone and Joint Surgery. British volume, 87(1), 108-110.

12. King, R. J., \& Hoyes, A. D. (1982). The mirror hand abnormality. The Hand, 14(2), 188-193.

13. Pintilie, D., Hatmanu, D., Olaru, I., \& Panoza, G. (1964). Double ulna with symmetrical polydactyly. The Journal of bone and joint surgery. British volume, 46(1), 89-93.

14. Yang, S. S., Jackson, L., Green, D. W., \& Weiland, A. J. (1996). A rare variant of mirror hand: a case report. The Journal of hand surgery, 21(6), 1048-1051.

15. De Smet, L. (1999). Ulnar dimelia. Acta orthopaedica belgica, 65(3), 382.

16. Gorriz, G. (1982). Ulnar dimelia-a limb without anteroposterior differentiation. The Journal of Hand Surgery, 7(5), 466-469.

17. Chinegwundoh, J. O. M., Gupta, M., \& Scott, W. A. (1997). Ulnar Dimelia: Is it a true duplication of the ulna? Journal of Hand Surgery, 22(1), 77-79.

18. Muradian, A. A. (2007). Ulnar dimelia, a case report. Basrah Journal of Surgery, 13(2), 73-74.

19. Apiou, F., Flagiello, D., Cillo, C., Malfoy, B., Poupon, M. F., \& Dutrillaux, B. (1996). Fine mapping of human HOX gene clusters. Cytogenetic and Genome Research, 73(1-2), 114-115.

20. Matsumoto, N., Ohashi, H., Kato, R., Fujimoto, M., Tsujita, T., Sasaki, T., ... \& Niikawa, N. (1997). Molecular mapping of a translocation breakpoint at $14 \mathrm{q} 13$ in a patient with mirror-image polydactyly of hands and feet. Human genetics, 99(4), 450-453.

21. Hussl, C. H. H. (1999). A case of mirror hand deformity with a 17-year postoperative follow up: case report. Scandinavian journal of plastic and reconstructive surgery and hand surgery, 33(3), 329-333.

22. Tsuyuguchi, Y., Tada, K., \& Yonenobu, K. (1982). Mirror hand anomaly: reconstruction of the thumb, wrist, forearm, and elbow. Plastic and Reconstructive Surgery, $70(3), 384-387$. 